\title{
Propriedades Psicométricas e Adaptação Cultural da Basic Need Satisfaction in General Scale para uma População Brasileira de Usuários de CADEIRA de Rodas ${ }^{1,2}$ \\ Psychometric Properties and Cultural Adaptation of the Basic NeED Satisfaction in GENERAL SCALE FOR a Brazilian Population of WheElCHair USERS
}

\author{
Valdilene WAGNER ${ }^{3}$ \\ Evandro Morais PEIXOTO ${ }^{4}$ \\ Leonardo Pestillo de OLIVEIRA ${ }^{5}$
}

\begin{abstract}
RESUMO: Este estudo teve como objetivo a adaptação cultural e a validação da Basic Need Satisfaction in General Scale (BNSG) para a sua aplicação no contexto cultural brasileiro. Participaram da pesquisa 133 pessoas com deficiência física, usuárias de cadeira de rodas. A adaptação cultural passou por um grupo focal e por um linguista da área de Letras. Os métodos estatísticos foram sistematicamente empregados para atender aos objetivos de adaptação cultural, estimativa de evidências de validade baseada no conteúdo, na estrutura interna, na relação com variável externa e na precisão para a versão brasileira da BNSG. A Análise Fatorial Exploratória indicou a adequação do modelo composto por um fator com bons indicadores de precisão, coeficiente Alpha de Cronbach igual a 0,916, e ômega de McDonald igual a 0,916. Os resultados desta pesquisa asseguram a adequação do processo de adaptaçáo cultural da $B N S G$ para o contexto brasileiro, resultando em uma nova ferramenta para pesquisas relacionadas a necessidades psicológicas básicas compreendidas no cenário da teoria da autodeterminação.
\end{abstract}

PALAVRAS-CHAVE: Psicometria. Necessidades psicológicas básicas. Autodeterminação. Pessoa com deficiência.

ABSTRACT: This study aimed at the cultural adaptation and validation of the Basic Need Satisfaction in General Scale (BNSG) for its application in the Brazilian cultural context. The participants of research were 133 people with physical disabilities, wheelchair users. The cultural adaptation involved a focus group and a linguist from the field of Portuguese Language Degree. Statistical methods were systematically employed to meet the objectives of cultural adaptation, estimation of evidence of validity based on content, internal structure, relation with external variable and accuracy for the Brazilian version of the BNSG. The Exploratory Factorial Analysis indicated the adequacy of the model composed of a factor with good indicators of accuracy, Cronbach's Alpha coefficient equal to 0.916, and McDonald's omega equal to 0.916. The results of this research assure the adequacy of the BNSG cultural adaptation process for the Brazilian context, resulting in a new tool for research related to basic psychological needs included in the scenario of the theory of self-determination.

KEYWORDS: Psychometry. Basic Psychological Needs. Self-determination. Person with Disability.

\section{INTRODUÇÃo}

Em países em desenvolvimento, estima-se que pelo menos $2 \%$ das pessoas, o que equivale a 121.800.000, necessitam de cadeira de rodas (Wheelchair Foundation, 2020). No

\footnotetext{
${ }^{1}$ https://doi.org/10.1590/1980-54702021v27e0214

${ }^{2}$ Agradecemos à Coordenaçấo de Aperfeiçoamento de Pessoal de Nível Superior (CAPES) pelo apoio financeiro para desenvolver este estudo.

${ }^{3}$ Mestre em Promoção da Saúde pela Universidade Cesumar. Maringá / Paraná / Brasil. E-mail: valdilenewagner1@gmail.com. ORCID: https://orcid.org/0000-0003-2558-7832

${ }^{4}$ Doutor em Psicologia. Docente do Programa de Pós-Graduação da Universidade São Francisco. Campinas / São Paulo / Brasil. E-mail: peixotoem@gmail.com. ORCID: https://orcid.org/0000-0003-1007-3433

${ }^{5}$ Doutor em Psicologia Social. Docente do Programa de Pós-Graduação da Universidade Cesumar. Maringá / Paraná / Brasil. E-mail: leopestillo@gmail.com. ORCID: https://orcid.org/0000-0001-5278-0676
} 
entanto, fazer pesquisa com esse grupo, no Brasil, é um grande desafio. Isso porque os sistemas públicos e privados de saúde e educação não possuem redes unificadas de catalogação por tipo de deficiência, o que aumenta a invisibilidade. Além disso, os projetos de fomento científicos e tecnológicos em saúde, direcionados para esses grupos, ocorrem com baixa frequência quando comparados à população em geral (Kang et al., 2016; Machado et al., 2018; Ursine et al., 2018). Por isso, a adaptação cultural da Basic Need Satisfaction in General Scale (BNSG) com brasileiros usuários de cadeira de rodas poderá facilitar estudos capazes de reduzir as lacunas relacionadas à saúde mental dessas pessoas, minimizando os danos provocados pela invisibilidade social imposta pelas já referidas circunstâncias.

As crises em países de baixa e média renda, como o Brasil, têm atingido vários setores, passando pela economia, pelas civilizaçóes, pelo meio ambiente, pelo pensamento, pela educação, pela demografia e por diversas outras esferas sociais, afetando diretamente as pessoas com deficiência, tornando-as mais vulneráveis pela precariedade de serviços públicos, pela debilidade econômica, pela insegurança e pelas violentas divergências políticas. Para obter, manter ou melhorar a qualidade de vida frente a essa realidade paradoxal, muitos indivíduos buscam o controle da própria existência de maneira autônoma e independente. A teoria da autodeterminação (Deci \& Ryan, 2000) argumenta que esse envolvimento autorregulado, em qualquer atividade, necessita da satisfação de três necessidades psicológicas inatas: autonomia, competência e relaçóes de pertencimento, as quais estão relacionadas à saúde, à motivação intrínseca, ao engajamento, à proatividade e ao bem-estar, com potencial para serem uma inusitada alternativa para que as pessoas com deficiência tenham possibilidades de deliberar, também, em situaçóes de crise (Bakhshi et al., 2018; Rohwerder, 2015; Vansteenkiste \& Ryan, 2013).

A teoria da autodeterminação dialoga com elementos psíquicos intersubjetivos que são integrados de maneira a motivar indivíduos para o estabelecimento de relações de interação em um ambiente social. Antagonicamente, o ambiente pode satisfazer as necessidades psicológicas básicas, mas também pode frustrá-las ou privá-las (Ryan \& Deci, 2009). Um ambiente favorável à satisfação das necessidades psicológicas básicas pode contribuir com o desenvolvimento pleno das potencialidades do indivíduo, sendo este autônomo para escolher e realizar suas vontades com liberdade, competente para almejar e atingir os objetivos desejados, e pertencente a outros grupos em um processo autêntico de interação e de reconhecimento social (Vansteenkiste \& Ryan, 2013).

No entanto, o modelo médico da deficiência, fortemente questionado no final do século XX, igualou a ideia de deficiência à ideia de incapacidade, atrelando ao indivíduo características puramente funcionais. Nessa perspectiva, um corpo com deficiência seria um corpo fisicamente incapaz, corroborando assim a criação de estigmas que culminariam em situaçóes de desvantagens sociais, privando e/ou frustrando muitos anseios dos indivíduos com deficiência (França, 2013). Opondo-se a essas frustraçóes, um ambiente favorável à satisfação das necessidades psicológicas pode proporcionar aos indivíduos maiores sentimentos de bem-estar geral e saúde mental (Vansteenkiste et al., 2020). Essas afirmaçóes foram validadas em variados contextos e em distintas áreas. Dentre elas, encontram-se os estudos associados à saúde (Britton et al., 2014), à educação (Lynch \& Salikhova, 2016) e ao comportamento humano (Martela \& Ryan, 2016). 
Dada a relevância dos debates feitos sobre a autodeterminação, foram pensados instrumentos para amplificar descobertas correspondentes à teoria. Dentre eles, tem-se a BNSG (Deci \& Ryan, 1985, 2002; Ryan \& Deci, 2009), a qual teve origem a partir da adaptação da Basic Needs Satisfaction at Work Scale (Gagné, 2003; Johnston \& Finney, 2010) e foi validada, também, em Portugal por Sousa et al. (2012).

Desde então, as pesquisas sobre satisfação das necessidades psicológicas básicas vêm recebendo atenção de estudiosos de diversas e distintas áreas em diferentes países do globo. Em vista disso, pesquisas e teorias relacionadas ao bem-estar estão sendo realizadas com abordagens variadas. A teoria da autodeterminação tem sido considerada necessária para determinar fatores específicos do bem-estar humano relacionado às necessidades psicológicas básicas e tem integrado, ocasionalmente, populaçóes com deficiência (Akkerman et al., 2018). Contudo, no Brasil, ainda não existem instrumentos cujas propriedades psicométricas sejam reconhecidas por pesquisadores ou por profissionais atuantes nessa área. Essa ausência acarreta prejuízos aos grupos vulneráveis, como de pessoas com deficiência, pois um instrumento capaz de mensurar as necessidades psicológicas é essencial para que sejam feitas novas descobertas sobre possibilidades de melhoria do bem-estar, da saúde e da qualidade de vida dessa população.

Objetivou-se, portanto, realizar a adaptação cultural e as análises das propriedades psicométricas da BNSG em uma amostra brasileira de pessoas com deficiência, usuárias de cadeira de rodas. Assim sendo, proporcionou-se o desenvolvimento de estudos futuros que podem, inclusive, sugerir possibilidades de redução de fatores de risco derivados de crises em diversos setores da sociedade brasileira e que atingem intensivamente grupos vulneráveis com deficiência.

\section{Método}

A metodologia deste estudo foi pensada de modo a dar suporte e complementar o objetivo da pesquisa. Dessa maneira, objetivou-se descrever o processo de adaptação cultural e de validação do instrumento para o Brasil da Basic Need Satisfaction in General Scale (BNSG), de validação portuguesa realizada por Sousa et al. (2012).

\subsection{Tipo De Pesquisa}

Este estudo caracteriza-se como metodológico e obedece aos procedimentos internacionais recomendados pela literatura. Esse tipo de pesquisa investiga os métodos de obtenção, organização e análise dos dados, tratando da elaboração, validação e avaliação de instrumentos (Prodanov \& Freitas, 2013).

\subsection{Participantes}

A amostra foi composta por 133 adultos com deficiência física, usuários de cadeira de rodas, selecionados de forma aleatória da população em geral. A maioria do público foi caracterizada pelo sexo masculino, por solteiros e com o Ensino Médio completo. 


\subsection{INSTRUMENTO}

A BNSG tem como base a teoria da autodeterminação originalmente desenvolvida por Deci e Ryan (2000). Como aspecto positivo, tem-se a paridade entre o Português de Portugal e o Português do Brasil. Por náo apresentarem diferenças capazes de prejudicar o levantamento e a análise dos dados dessa pesquisa, a adaptaçáo cultural do instrumento tornou-se eficaz. Para realização da adaptação cultural, é necessário que se sigam algumas fases que contemplam as diretrizes desse processo. A escala original utilizada foi a BNSG. Por conter validação para o Português de Portugal elaborado por Sousa et al. (2012) do instrumento desenvolvido por Deci e Ryan (2000), optou-se por uma validação e adaptação cultural na forma linguística para o português do Brasil. O instrumento da $B N S G^{6}$ é composto por 21 itens, com uma escala de resposta tipo Likert de sete pontos, dividido por três fatores, que correspondem às três necessidades psicológicas básicas: (a) com sete itens no fator Autonomia (itens $1,4^{*}$, $8,11^{*}, 14,17,20^{*}$ ); (b) com seis itens no fator Competência (itens $3^{*}, 5,10,13,15^{*}, 19^{*}$ ); e (c) oito itens no fator Relaçóes de Pertença (itens 2, 6, 7*, 9, 12, 16*, 18*, 21). Os itens marcados com asterisco $\left.{ }^{*}\right)$ devem ter suas pontuaçóes invertidas no momento da correçáo. $\mathrm{O}$ instrumento tem flexibilidade para ser utilizado com as três dimensóes agregadas ou com cada uma das dimensões de forma independente. Neste estudo, as análises indicaram a utilização do instrumento como unidimensional.

\subsection{Procedimentos}

O estudo foi aprovado pelo Comitê de Ética em Pesquisa por meio do Parecer de número 2.503.634, do Centro Universitário de Maringá (UniCesumar), Paraná, Brasil. A utilização do instrumento foi devidamente autorizada por Edward Deci e Richard Ryan e pelos responsáveis pela validação da versão do instrumento em Portugal, representados pelos coautores António Labisa Palmeira, da ULisboa, e José Luís Pais Ribeiro, da U.Porto.

\subsection{TraduÇão E ADAPTAÇáo}

A tradução e a adaptação cultural da versão do instrumento validado em Portugal passaram por uma juíza da área de Letras, linguista e especialista em análise de discurso que supervisionou a tradução, a adaptação e a validação do conteúdo. Depois de finalizar a validação do conteúdo, realizou-se um estudo piloto com um grupo focal composto por sete pessoas adultas com características idênticas aos sujeitos da pesquisa. Dois encontros foram realizados com o grupo focal. No primeiro, foi apresentada a proposta, a finalidade e o método utilizado. Posteriormente, os participantes que concordaram com a participaçáo assinaram o Termo de Consentimento Livre e Esclarecido (TCLE). No segundo encontro, o grupo recebeu as questóes que compóem o instrumento. Foi feita a leitura de cada uma das questóes em voz alta. Depois, os membros do grupo avaliaram a qualidade das perguntas do instrumento e a coerência da linguagem e do conteúdo. Esse processo foi gravado, transcrito e encaminhado à juíza da área de Letras, linguista e especialista em análise de discurso. A juíza reavaliou a gramática da tradução; as alteraçóes semânticas foram feitas após o grupo focal. Uma síntese foi realizada e obteve-se uma versão final das traduçóes.

\footnotetext{
${ }^{6}$ Ver a versão brasileira da BNSG ao final deste texto.
} 


\subsection{ANÁLISE DE DADOS}

Os métodos estatísticos, utilizados neste estudo, foram sistematicamente apresentados para atender-se ao objetivo de estimativa de evidências de validade, com base na estrutura interna e de precisão. Para tanto, diferentes métodos estatísticos foram empregados: Análise Paralela (AP) e Análise Fatorial Exploratória (AFE). A AP é considerada um dos procedimentos mais adequados para determinar o número de fatores a serem retidos. Consiste em um conjunto hipotético de comparação de matrizes de correlação de variáveis utilizando a mesma dimensionalidade como base do conjunto de dados reais. A AP foi utilizada como critério para determinar o número correto de fatores a serem retirados. Neste estudo, sugeriu-se que poderia ser feita com três fatores (Lorenzo-Seva, 2011).

A partir dos indicadores da AP realizou-se a AFE com o método de estimação Robust Diagonally Weighted - Robust Promin (Lorenzo-Seva \& Ferrando, 2019). Devido à natureza ordinal dos dados, a matriz de correlação policórica foi utilizada por meio do software estatístico Factor 10.10.1 (Ferrando \& Lorenzo-Seva, 2017). Uma das vantagens do programa Factor para a realização da AFE é a possibilidade de estimar índices clássicos de ajuste da análise fatorial confirmatória que indicam o nível de ajuste da solução fatorial aos dados analisados. Adicionalmente, em consideração ao tamanho da amostra, foram estimados valores de intervalo de confiança por meio dos métodos de reamostragem por bootstrap, com mais especificamente 500 matrizes de correlação estimadas aleatoriamente. Os índices de ajuste considerados foram: $\chi 2$ (qui-quadrado); razão $\chi 2 / g 1$; Comparative Fit Index (CFI); Non-Normed Fit Index (NNFI); Goodness of Fit Index (GFI); Root-Mean-Square Error of Approximation (RMSEA); e Bayesian Information Criterion (BIC). A avaliação da consistência interna ocorreu pelo cálculo dos índices Alpha de Cronbach e do cálculo Ômega de McDonald.

\section{Resultados}

Esta seção apresenta os resultados relacionados a três elementos: características sociodemográficas dos participantes; estrutura e consistência interna do instrumento BNSG; e evidência de validade baseada na relação com variável externa.

\subsection{CARACTERÍSTICAS SOCIODEMOGRÁFICAS DOS PARTICIPANTES}

O perfil sociodemográfico demonstrou que os participantes com deficiência física, usuários de cadeira de rodas, tinham uma média de idade de 34,37 anos e eram, em sua maioria, do sexo masculino (69,17\%); as mulheres representavam 30,83\% da amostra. Mais da metade dos participantes eram solteiros $(58,64)$. Em relação à escolaridade, apenas 15,04\% possuíam Ensino Superior completo. Dos participantes, 33,08\% são aposentados e um número expressivo de participantes pratica exercício físico (79,05\%). As características demográficas completas são apresentadas na Tabela 1 a seguir. 


\section{Tabela 1}

Perfil sociodemográfico das pessoas com deficiência fisica, usuárias de cadeira de rodas

\begin{tabular}{ll}
\hline \multicolumn{1}{c}{ Variáveis } & \multicolumn{1}{c}{ X (DP) } \\
\hline Idade & $34,37(9,22)$ \\
Sexo & $\mathrm{N}(\%)$ \\
Masculino & $92(69,17)$ \\
Feminino & $41(30,83)$ \\
Estado Civil & $\mathrm{N}(\%)$ \\
Solteiro(a) & $78(58,64)$ \\
Casado(a) & $32(24,06)$ \\
União estável & $18(13,54)$ \\
Divorciado(a) & $3(2,26)$ \\
Viúvo(a) & $2(1,50)$ \\
Educação Básica e Educação Superior & $\mathrm{N}(\%)$ \\
Ensino Fundamental incompleto & $14(10,53)$ \\
Ensino Fundamental completo & $6(4,51)$ \\
Ensino Médio incompleto & $17(12,78)$ \\
Ensino Médio completo & $42(31,58)$ \\
Ensino Superior incompleto & $25(18,80)$ \\
Ensino Superior completo & $20(15,04)$ \\
Pós-Graduação & $9(6,76)$ \\
Aposentado(a) & $44(33,08)$ \\
Prática de Exercício & $105(79,05)$ \\
\hline
\end{tabular}

Nota. DP - Desvio padrão.

\subsection{ESTRUTURA E CONSISTÊNCIA INTERNA DO INSTRUMENTO BASIC NEED SATISFACTION IN GENERAL SCALE (BNSG)}

A primeira análise realizada no instrumento para verificar sua viabilidade de utilização foi a AP. A priori, essa análise sugeriu a possibilidade de reter-se dois fatores para a realizaçáo da AFE, haja vista que apenas os dois primeiros apresentaram percentagem de variância explicada (39.68 e 11.94, respectivamente) superior aos valores de variância explicada alocado no percentil de 95 para as matrizes estimadas por meio dos dados aleatórios (11.51 e 10.24, respectivamente).

Com base nesses resultados, foram avaliados os modelos unifatoriais, compostos por dois fatores. Os índices de ajuste de ambos os modelos são apresentados na Tabela 2 que segue. Os resultados da Tabela 2 permitem verificar os índices de adequação aceitáveis ao modelo unidimensional e os índices classificados como bons para o modelo composto por dois fatores. 
Adicionalmente, o valor de $B I C$ menor para o segundo modelo sugere que este deve ser o modelo preferido (Kline, 2011).

\section{Tabela 2}

Índices de ajustamento do modelo e adequação da amostra

\begin{tabular}{lll}
\hline & $\boldsymbol{B N S G} \mathbf{1}$ fator & $\boldsymbol{B N S G} 2$ fatores \\
\hline KMO & $0.78225(0.789-0.792)$ & \\
Bartlett (Df)/P-value & $1441,0(210) /<0.001$ & \\
CFI & $0.942(0.888-0.986)$ & $0.986(0.968-1.00)$ \\
GFI & $0.919(0.870-0.967)$ & $0.986(0.968-1.00)$ \\
NNFI & $0.936(0.876-0.985)$ & $0.982(0.960-1.00)$ \\
RMSEA & $0.091(0.062-0.104)$ & $0.043(0-0.056)$ \\
BIC & $606.798(483.154-669.014)$ & $519.391(467.784-549.372)$ \\
\hline
\end{tabular}

Nota. Devido à limitação do tamanho da amostra, foram estimados intervalos de Confiança de 95\% dos índices de ajuste avaliados. Os valores apresentados entre parênteses são provenientes de 500 matrizes de correlação estimadas aleatoriamente.

$\mathrm{KMO}$ - critério de Kaiser-Meyer-Olkin: identifica se um modelo de análise fatorial está adequadamente ajustado aos dados.

Com isso, o próximo passo foi verificar as cargas fatoriais. O modelo fatorial de dois fatores é apresentado na Tabela 3 em que são postos os resultados referentes aos modelos de um e de dois fatores.

\section{Tabela 3}

Modelo fatorial da Escala de Satisfação das Necessidades Básicas - modelos de um e de dois fatores

\begin{tabular}{lllll}
\hline & \multicolumn{2}{c}{ Unifatorial } & & \multicolumn{2}{c}{ Dois fatores } \\
\cline { 1 - 2 } $\mathbf{1}$ & \multicolumn{1}{c}{ F1 } & & F1 & F2 \\
2 & 0.528 & & 0.323 & \\
$\mathbf{3}$ & 0.684 & 0.694 & \\
$\mathbf{4}$ & 0.567 & & 0.714 \\
$\mathbf{5}$ & 0.403 & & 0.479 \\
$\mathbf{6}$ & 0.596 & 0.527 & \\
$\mathbf{7}$ & 0.745 & 0.908 & \\
$\mathbf{8}$ & 0.560 & & 0.708 \\
$\mathbf{9}$ & 0.564 & 0.546 & \\
$\mathbf{1 0}$ & 0.600 & 0.790 & \\
$\mathbf{1 1}$ & 0.702 & 0.672 & \\
$\mathbf{1 2}$ & 0.333 & & 0.511 \\
$\mathbf{1 3}$ & 0.525 & 0.863 &
\end{tabular}




\begin{tabular}{llll}
$\mathbf{1 4}$ & 0.589 & 0.777 & \\
$\mathbf{1 5}$ & 0.553 & & 0.750 \\
$\mathbf{1 6}$ & 0.500 & & 0.792 \\
$\mathbf{1 7}$ & 0.708 & 0.671 & \\
$\mathbf{1 8}$ & 0.629 & & 0.616 \\
$\mathbf{1 9}$ & 0.694 & & 0.769 \\
$\mathbf{2 0}$ & 0.639 & & 0.613 \\
$\mathbf{2 1}$ & 0.542 & 0.469 & \\
\hline Corr. & & 0.579 & \\
\hline E.V & 37.2 & 38.13 & 11.68 \\
Total E.V & & 49.81 & \\
\hline Alpha & 0.916 & 0.908 & 0.901 \\
Ômega & 0.916 & 0.907 & 0.903 \\
\hline
\end{tabular}

Nota . Corr. = correlação; E.V - Explained variance.

São consideradas cargas fatoriais as associações entre os itens e os fatores. Quanto maior a carga fatorial, maiores são as possibilidades de correlação com um determinado fator. $\mathrm{Na}$ interpretação dos fatores, podem ser consideradas cargas fatoriais estatisticamente importantes aquelas acima de 0,3 (Field, 2013). Nesse sentido, considera-se a adequação de todos os itens dos instrumentos para ambos os fatores, uma vez que todos apresentaram cargas superiores a 0.333 (modelo unidimensional) e 0,323 (modelo de dois fatores).

Contudo, ao interpretar teoricamente o agrupamento dos itens, observou-se uma incongruência no modelo de dois fatores, haja vista que esse agrupamento pode ocorrer em função dos itens negativos e positivos e, portanto, não permitia uma interpretação adequada dos fatores com base no conteúdo dos itens. Ressalta-se que os itens negativos foram invertidos antes da realização das análises. Dessa forma, optou-se pela adoção do modelo unidimensional, que contou com itens com cargas fatoriais adequadas, caracterizando-se com bons representantes do construto avaliados pelo fator. Adicionalmente, o modelo foi capaz de explicar $37,2 \% \mathrm{da}$ variância total dos dados disponíveis.

Para avaliar a precisão da BNSG, utilizou-se um conjunto de indicadores (Alpha de Cronbach e Ômega de McDonald), com coeficientes acima de 0,7 considerados aceitáveis (P. A. Kline, 2015). Verifica-se ainda na Tabela 3 que o modelo unidimensional apresentou boas evidências de precisão.

\section{3 EVIDÊNCIA DE VALIDADE BASEADA NA RELAÇÃO COM VARIÁVEL EXTERNA}

Foi realizada validação externa utilizando a $B N S G$ e o WHOQOL-bref (World Health Organization Quality of Life), instrumento de avaliação da qualidade de vida. As análises demonstraram que as correlaçóes foram significativas ( $<<0,001, \mathrm{p}<0,01)$. Os resultados da correlação são apresentados na Tabela 4 que segue. 


\section{Tabela 4}

Correlação entre variáveis de qualidade de vida e satisfação de necessidades básicas

\begin{tabular}{|c|c|c|c|c|c|}
\hline & \multicolumn{4}{|c|}{ Variáveis Qualidade de Vida (WHOQOL) } & \multirow{2}{*}{ BNSG } \\
\hline & Físico & Psicológico & Relaçóes Sociais & Meio Ambiente & \\
\hline Físico & - & & & & \\
\hline Psicológico & $0.893^{* * *}$ & - & & & \\
\hline Relaçôes Sociais & $0.784^{* * *}$ & $0.893^{* * *}$ & - & & \\
\hline Meio Ambiente & $0.849^{* * *}$ & $0.833^{* * *}$ & $0.771^{* * *}$ & - & \\
\hline BNSG & $0.622^{* * *}$ & $0.656^{* * *}$ & $0.603^{* * *}$ & $0.565^{* * *}$ & - \\
\hline
\end{tabular}

Observação: ${ }^{* * *} \mathrm{p}<0.001 ;{ }^{* *} \mathrm{p}<0.01$.

\section{Discussāo}

Este é o primeiro estudo a validar e a adaptar culturalmente a BNSG da teoria de autodeterminação para o contexto brasileiro. Um estudo de Nascimento Junior et al. (2018) traduziu e adaptou a $B N S G$ para o Brasil, mas, exclusivamente, para a versáo específica para a área do esporte. Além disso, pela primeira vez foram comprovadas evidências psicométricas dessa escala em uma amostra de pessoas com deficiência física, usuárias de cadeira de rodas. A BNSG teve origem, como já afirmado, na adaptação da Basic Needs Satisfaction at Work Scale (Gagné, 2003; Johnston \& Finney, 2010). Johnston e Finney (2010) analisaram pela primeira vez a estrutura fatorial do instrumento e optaram pela análise fatorial confirmatória, testando um e três fatores. De um modo geral, esse estudo mostrou que o modelo composto por dois fatores para a $B N S G$ apresentou índices de ajustes ligeiramente superiores ao modelo unifatorial. Contudo, a organização dos itens não permitia interpretação sustentada teoricamente, uma vez que ela representava apenas uma separação dos itens em função da ordenação da escala de resposta (Lindwall et al., 2012).

Esse fenômeno tem sido discutido exaustivamente na literatura especializada, e, dentre as discussóes, encontra-se o apontamento de que a utilização de itens invertidos pode levar à composição de um fator de método, resultando em estrutura fatorial não esperada teoricamente (Distefano \& Motl, 2006). Um exemplo prático dessa discussão é a Escala de Autoestima de Rosemberg (1989), instrumento que conta com itens positivos e negativos. Estudos divergem quanto à estrutura interna desse instrumento. Alguns a defendem como uma medida unidimensional, e outros como uma medida de dois fatores, as quais se diferem por agruparem os itens positivos e negativos, e, portanto, representaria um artefato da redação dos itens (Greenberg et al., 2002). Dessa forma, na presente pesquisa, optou-se pela estrutura interna que apresentava melhor possibilidade de interpretação teórica do agrupamento de itens.

A versão traduzida e adaptada apresentou valores de confiabilidade adequados, como apontam Nunnally \& Bernstein (1994) e Kline (2015). O modelo fatorial demonstrou que todos os fatores poderiam ser considerados. Contudo, na Análise Fatorial Exploratória com três fatores, os itens não se ajustaram corretamente aos fatores correspondentes. A análise com dois fatores não encontrou coerência entre o conteúdo dos itens para dois fatores distintos. Por isso, optou-se por fazer uma análise unidimensional que revelou consistência interna e bons índices de ajuste. Nesse 
estudo, considerou-se 0.4 como carga fatorial ideal por item. Com isso, dois itens precisavam passar por análise cuidadosa do conteúdo: o item 4 (0,328) e o item 11 (0,286). Assim, constata-se que podem ocorrer estudos em que esses itens possam ser excluídos. Todos os outros itens tiveram cargas satisfatórias, conferindo boa adequação do modelo unidimensional.

No que concerne à confiabilidade interna de um instrumento, esta é a capacidade de um instrumento produzir resultados confiáveis em diferentes situações. Foram medidas a confiabilidade com consistência interna para avaliar o grau em que todos os itens do instrumento se referem ao mesmo sujeito e a estabilidade temporal para avaliar a variação do tempo no instrumento.

O Alpha de Cronbach é uma estimativa de confiabilidade bastante comum em testes de linguagem. É utilizado para aferir a consistência interna, por fornecer uma estimativa segura da confiabilidade de um conjunto de resultados de testes. É interpretado a partir da estimativa da proporção de variância sistemática ou consistente em um conjunto de pontuaçóes do teste. Tal qual o Alpha de Cronbach, o Ômega de McDonald é um estimador de confiabilidade. O Ômega tem um índice bastante sensível de consistência interna, tanto em relação ao Alpha quanto em comparação a outras possibilidades. Os valores de Alpha e Ômega são boas alternativas de confiabilidade na análise de consistência interna, portanto aplicáveis em escalas psicométricas (Zinbarg et al., 2005). Para o estudo em questão, os valores foram considerados adequados tanto para o Alpha quanto para o Ômega.

Uma possível limitação para este estudo esteve relacionada ao tamanho da amostra, composta por pessoas com deficiência física, usuárias de cadeira de rodas, em sua maioria do sexo masculino. Essa amostra fornece possibilidades limitadas para análise fatorial com três e dois fatores por representar, de maneira restritiva, a população geral do público-alvo. Ademais, a ausência de catalogação dessa população, pelos setores sociais de saúde e educação, precarizaram a robustez do banco de dados. Por isso, estudos futuros devem atentar-se às questóes relativas a esses resultados. Contudo, escolheu-se por população da pesquisa as pessoas com deficiência física, usuárias de cadeira de rodas, por tratar-se de um grupo vulnerável ainda carente de pesquisas científicas e que representam uma parcela significativa da população brasileira.

Outros estudos realizados na Bélgica, na China, nos Estados Unidos, no Japão e no Peru, com populações diversas, também confirmam que a satisfação das necessidades psicológicas básicas são elementos fundamentais para a saúde e o bem-estar, confirmando sua importância e aplicação em diferentes culturas (Nishimura \& Suzuky, 2016). A BNSG tem sido adaptada e utilizada em diversos domínios específicos, como educação, relacionamentos, esportes, ambientes de trabalho e cuidados médicos (Deci \& Ryan, 2000). Assim sendo, sua validação e adaptação cultural tornou-se importante, também, para o contexto brasileiro.

\section{Conclusóes}

O modelo unidimensional da escala apresentou boas propriedades psicométricas. Há uma limitação em relação à análise de três e de dois fatores, possivelmente em decorrência do número da amostra. Estudos futuros precisam avançar em relação a essa área do conhecimento para identificar contextos que suportam versus frustram as necessidades psicológicas básicas e que impactam diretamente no bem-estar psicológico, relacionado à saúde mental das pessoas 
com deficiência, oportunizando a elas acesso aos domínios da qualidade de vida, da autodeterminação e do reconhecimento de suas identidades.

\section{REFERÊNCIAS}

Akkerman, A., Kef, S., \& Meininger, H. P. (2018). Job satisfaction of people with intellectual disabilities: the role of basic psychological need fulfillment and workplace participation. Disability and Rehabilitation, 40(10), 1192-1199. https://doi.org/10.1080/09638288.2017.1294205

Bakhshi, P., Babulal, G. M., \& Trani, J. F. (2018). Education and disability in a conflict affected context: Are children with disabilities less likely to learn and be protected in Darfur? World development 106, 248-259. https://doi.org/10.1016/j.worlddev.2018.01.019

Britton, P. C., Van Orden, K. A., Hirsch, J. K., \& Williams, G. C. (2014). Basic psychological needs, suicidal ideation, and risk for suicidal behavior in young adults. Suicide \& life-threatening behavior, 44(4), 362-371. https://doi.org/10.1111/sltb.12074

Deci, E. L., \& Ryan, R. M. (1985). Intrinsic motivation \& self-determination in human behavior. Plenum.

Deci, E. L., \& Ryan, R. M. (2000). The "what" and "why" of goal pursuits: human needs and the self-determination of behavior. Psychological Inquiry, 11(4), 227-268. https://doi.org/10.1207/ S15327965PLI1104_01

Deci, E. L., \& Ryan, R. M. (2002). Handbook of self-determination research. University of Rochester Press.

Distefano, C., \& Motl, R. W. (2006). Further investigating method effects associated with negatively worded items in self-report surveys. Structural Equation Modeling: A Multidisciplinary Journal, 13(3), 440-64. https://doi.org/10.1207/s15328007sem1303_6

Ferrando, P. J., \& Lorenzo-Seva, U. (2017). Program FACTOR at 10: origins, development and future directions. Psicothema, 29(2), 236-241. https://doi.org/10.7334/psicothema2016.304

Field, A. (2013). Discovering Statistics Using IBM SPSS Statistics. Sage.

França, T. H. (2013). Modelo social da deficiência: uma ferramenta sociológica para a emancipação social. Lutas Sociais, 17(31), 59-73.

Gagné, M. (2003). The role of autonomy support and autonomy orientation in prosocial behavior engagement. Motivation and Emotion, 27, 199-223. https://doi.org/10.1023/A:1025007614869

Greenberger, E., Chuansheng, C., Dmitrieva, J. \& Farruggia, S. (2002). Item-wording and the dimensionality of the Rosenberg Self-Esteem Scale: do they matter? Personality and Individual Differences, 35, 1241-1254. https://doi.org/10.1016/S0191-8869(02)00331-8

Johnston, M. M., \& Finney, S. J. (2010). Measuring basic needs satisfaction: Evaluating previous research and conducting new psychometric evaluations of the Basic Needs Satisfaction in General Scale. Contemporary Educational Psychology, 35(4), 280-296. https://doi.org/10.1016/j. cedpsych.2010.04.003

Kang, Q., Chen, G., Lu, J., \& Yu H. (2016). Health disparities by type of disability: health examination results of adults (18-64 years) with disabilities in Shanghai, China. PloS one, 11(5). https://doi. org/10.1371/journal.pone.0155700

Kline, P. A. (2015). Handbook of Test Construction: Introduction to Psychometric Design. Routledge Press. 
Kline, R. (2011). Principles and Practice of Structural Equation Modeling.

Lindwall, M., Barkoukis, V., Grano, C., Lucidi, F., Raudsepp, L., Liukkonen, J., \& ThogersenNtoumani, C. (2012). Method effects: the problem with negatively versus positively keyed items', Journal of Personality Assessment, 94 (2), 196-204. https://doi.org/10.1080/00223891.2011.645936

Lorenzo-Seva, U. (2011). Horn's parallel analysis for selecting the number of dimensions in correspondence analysis. Methodology: European Journal of Research Methods for the Behavioral and Social Sciences, 7(3), 96-102. https://doi.org/10.1027/1614-2241/a000027

Lorenzo-Seva, U., \& Ferrando, P. J. (2019). Robust Promin: a method for diagonally weighted factor rotation. Technical report, URV.

Lynch, M. F., \& Salikhova, N. R. (2016). Teachers' conceptions about the child's developmental needs: a structural analysis. International Electronic Journal of Mathematics Education, 11(5), 1471-1479.

Machado, W. C. A., Pereira, J. de S., Schoeller, S. D., Júlio, L. C., Martins, M. M. F. P. da S., \& Figueiredo, N. M. A. de. (2018). Integralidade na rede de cuidados da pessoa com deficiência. Texto \& Contexto - Enfermagem, 27(3), 1-9. https://doi.org/10.1590/0104-07072018004480016

Martela, F., \& Ryan, R. M. (2016). The benefits of benevolence: basic psychological needs, beneficence, and the enhancement of well-being. Journal of Personality, 84(6), 750-764. https://doi.org/10.1111/ jopy. 12215

Nascimento Junior, J. R. A. do, Vissoci, J. R. N., \& Vieira, L. F. (2018). Propriedades Psicométricas da Versão Brasileira da Escala de Satisfação das Necessidades Básicas no Esporte (BNSSS). Psicologia: Teoria e Pesquisa, 34, e3456. https://doi.org/10.1590/0102.3772e3456

Nishimura, T., \& Suzuki, T. (2016). Basic Psychological Need Satisfaction and Frustration in Japan: Controlling for the Big Five Personality Traits. Japanese Psychological, 58(4), 320-331. https://doi. org/10.1111/jpr.12131

Nunnally, J. C., \& Bernstein, I. H. (1994) The assessment of reliability. Psychometric Theory, 3, 248-292.

Prodanov, C. C., \& Freitas, E. C. (2013). Metodologia do trabalho cientifico: métodos e técnicas da pesquisa e do trabalho acadêmico. Associação Pró-Ensino Superior em Novo Hamburgo Feevale.

Rohwerder, B. (2015). Disability inclusion: Topic guide. GSDRC, University of Birmingham.

Rosenberg, M. (1989). Society and the adolescent self-image. Wesleyan University Press.

Ryan, R. M., \& Deci, E. L. (2009). Promoting self-determined school engagement: Motivation, learning, and well-being. In K. R. Wenzel, \& A. Wigfield (Eds.), Educational psychology handbook series. Handbook of motivation at school (pp. 171-195). Routledge.

Sousa, S. S., Pais Ribeiro, J. L., Palmeira, A. L., Teixeira, P. J., \& Silva, M. N. (2012). Estudo da Basic Need Satisfaction in General Scale para a língua Portuguesa. Psicologia, Saúde \& Doença, 13(2), 209-219.

Ursine, B. L., Pereira, É. L., \& Carneiro, F. F. (2018). Saúde da pessoa com deficiência que vive no campo: o que dizem os trabalhadores da Atenção Básica? Interface - Comunicação, Saúde, Educação, 22(64), 109-120. https://doi.org/10.1590/1807-57622016.0666

Vansteenkiste, M., \& Ryan, R. M. (2013). On psychological growth and vulnerability: Basic Psychological Need Satisfaction and Need Frustration as a Unifying Principle. Journal of Psychotherapy Integration, 23(3), 263-280. 
Vansteenkiste, M., Ryan, R. M., \& Soenens, B. (2020). Basic psychological need theory: Advancements, critical themes, and future directions. Motivation and Emotion, 44, 1-31. https://doi.org/10.1007/ s11031-019-09818-1

Wheelchair Foundation. (2020). Wheelchair Needs In The World. https://www.wheelchairfoundation. org/fth/analysis-of-wheelchair-need/

Zinbarg, R. E., Revelle, W., Yovel, I., \& Li, W. (2005). Cronbach's $\alpha$, Revelle's $\beta$, and Mcdonald's $\omega$ H: their relations with each other and two alternative conceptualizations of reliability. Psychometrika, 70, 123-133. https://doi.org/10.1007/s11336-003-0974-7 


\section{APÊNDICE}

\section{Basic NeEd SatisfaCtion in GeNeral (BNSG) - Versáo Brasileira}

\section{O que sinto?}

Por favor, leia cuidadosamente cada uma das seguintes afirmaçôes, pense como se relacionam com a sua vida e indique se é verdadeira para você. Use a escala seguinte para responder:

\begin{tabular}{|c|c|c|c|c|c|c|c|c|}
\hline \multirow[b]{2}{*}{1} & \multirow[b]{2}{*}{$\begin{array}{l}\text { Sinto-me livre para decidir por mim mesmo/a como viver a } \\
\text { minha vida. }\end{array}$} & \multicolumn{2}{|c|}{$\begin{array}{c}\text { Nada } \\
\text { verdadeiro }\end{array}$} & \multicolumn{3}{|c|}{$\begin{array}{l}\text { Em parte, } \\
\text { verdadeiro }\end{array}$} & \multicolumn{2}{|c|}{$\begin{array}{l}\text { Totalmente } \\
\text { verdadeiro }\end{array}$} \\
\hline & & 1 & 2 & 3 & 4 & 5 & 6 & 7 \\
\hline 2 & Eu gosto das pessoas com quem convivo. & 1 & 2 & 3 & 4 & 5 & 6 & 7 \\
\hline 3 & Frequentemente, não me sinto muito competente. & 1 & 2 & 3 & 4 & 5 & 6 & 7 \\
\hline 4 & Sinto pressão na minha vida. & 1 & 2 & 3 & 4 & 5 & 6 & 7 \\
\hline 5 & $\begin{array}{l}\text { As pessoas que conheço me dizem que sou bom/a naquilo que } \\
\text { faço. }\end{array}$ & 1 & 2 & 3 & 4 & 5 & 6 & 7 \\
\hline 6 & Me dou bem com as pessoas que convivo. & 1 & 2 & 3 & 4 & 5 & 6 & 7 \\
\hline 7 & $\begin{array}{l}\text { Sou uma pessoa reservada e nâo tenho muitos relacionamentos } \\
\text { sociais. }\end{array}$ & 1 & 2 & 3 & 4 & 5 & 6 & 7 \\
\hline 8 & $\begin{array}{l}\text { Geralmente, sinto-me livre para expressar as minhas ideias e } \\
\text { opinióes. }\end{array}$ & 1 & 2 & 3 & 4 & 5 & 6 & 7 \\
\hline 9 & $\begin{array}{l}\text { Considero as pessoas com as quais convivo regularmente como } \\
\text { minhas amigas. }\end{array}$ & 1 & 2 & 3 & 4 & 5 & 6 & 7 \\
\hline 10 & Fui capaz de aprender recentemente coisas novas e interessantes. & 1 & 2 & 3 & 4 & 5 & 6 & 7 \\
\hline 11 & $\begin{array}{l}\text { Na minha vida diária tenho que fazer frequentemente o que me } \\
\text { mandam. }\end{array}$ & 1 & 2 & 3 & 4 & 5 & 6 & 7 \\
\hline 12 & $\begin{array}{l}\text { Sinto que as pessoas que me rodeiam no dia a dia se preocupam } \\
\text { comigo. }\end{array}$ & 1 & 2 & 3 & 4 & 5 & 6 & 7 \\
\hline 13 & Na maioria dos dias sinto-me realizado/a com aquilo que faço. & 1 & 2 & 3 & 4 & 5 & 6 & 7 \\
\hline 14 & $\begin{array}{l}\text { As pessoas com quem convivo no dia a dia costumam ter consi- } \\
\text { deraçáo por meus sentimentos. }\end{array}$ & 1 & 2 & 3 & 4 & 5 & 6 & 7 \\
\hline 15 & $\begin{array}{l}\text { Na minha vida náo tenho muitas condiçốes de demonstrar } \\
\text { aquilo que sou capaz. }\end{array}$ & 1 & 2 & 3 & 4 & 5 & 6 & 7 \\
\hline 16 & Não me sinto próximo de muitas pessoas. & 1 & 2 & 3 & 4 & 5 & 6 & 7 \\
\hline 17 & Sinto que posso ser eu mesmo/a no meu dia a dia. & 1 & 2 & 3 & 4 & 5 & 6 & 7 \\
\hline 18 & $\begin{array}{l}\text { As pessoas com quem convivo regularmente náo parecem gostar } \\
\text { muito de mim. }\end{array}$ & 1 & 2 & 3 & 4 & 5 & 6 & 7 \\
\hline 19 & Frequentemente não me sinto muito capaz. & 1 & 2 & 3 & 4 & 5 & 6 & 7 \\
\hline 20 & $\begin{array}{l}\text { Não tenho muitas oportunidades de decidir por mim mesmo/a } \\
\text { como fazer as coisas no meu dia a dia. }\end{array}$ & 1 & 2 & 3 & 4 & 5 & 6 & 7 \\
\hline 21 & Geralmente, as pessoas são muito simpáticas comigo. & 1 & 2 & 3 & 4 & 5 & 6 & 7 \\
\hline
\end{tabular}

Nota. Modelo Unidimensional - Informação para Cotação. Inversão dos escores das questóes: 3, 4, 7, 11, 15, 16, $18,19,20$. Para inverter a cotaçấo basta subtrair 8 à pontuação obtida na resposta ao item. ${ }^{7}$ 


\title{
Emergência de Respostas de Seguir InstruÇão e de Tato-Intraverbal APÓs INSTRUÇÁo COM MÚlTiPLOS EXEMPLARES ${ }^{1,2}$
}

\author{
EMERGENCE OF RULE FOLLOWING aNd TACT-INTRAVERBAL RESPONSES AFTER \\ MULTIPLE EXEMPLAR INSTRUCTION
}

\author{
Elaine de Carvalho SILVA ${ }^{3}$ \\ Nassim Chamel ELIAS ${ }^{4}$
}

\begin{abstract}
RESUMO: O objetivo deste estudo foi verificar os efeitos do ensino com Instrução com Múltiplos Exemplares (Multiple Exemplar Instruction - MEI), envolvendo, em cada tentativa, a resposta de seguir uma instrução tato-intraverbal para uma relação espacial (por exemplo, esquerda) na emergência de novas respostas e para uma relação espacial não ensinada diretamente (por exemplo, direita) para partes do corpo (braço, orelha, mão, perna). Adicionalmente, foi testada a generalização por meio de objetos que não foram utilizados no ensino com MEI. Os participantes foram quatro crianças e adolescentes diagnosticados com Transtorno do Espectro do Autismo (TEA) e deficiência intelectual, de sete a 14 anos. A sequência do procedimento foi: testes de seguir instrução e de tato-intraverbal em linha de base; ensino com $M E I$ para uma relação espacial; teste final de seguir instrução e de tato-intraverbal para a relação espacial não ensinada diretamente; teste de generalização. Foi utilizado o delineamento de múltiplas sondagens acoplado ao delineamento de linha de base múltipla entre os participantes. Os resultados sugerem que o ensino com $M E I$ foi suficiente para a emergência das respostas não ensinadas diretamente e de generalização. Pôde-se inferir que o procedimento foi eficaz e eficiente, pois gerou a emergência de respostas não ensinadas diretamente e a aprendizagem se deu em poucos blocos.
\end{abstract}

PALAVRAS-CHAVE: Linguagem. Instrução com Múltiplos Exemplares. Autismo.

\begin{abstract}
The objective of this study was to verify the effects of teaching with Multiple Exemplar Instruction (MEI) involving, in each trial, the response of following a tact-intraverbal instruction to a spatial relation (for example, left) in the emergence of new responses and for the spatial relation not directly taught (for example, right) for body parts (arm, ear, hand, leg). Additionally, generalization was tested by means of objects that were not used in teaching. Participants were four children and adolescents diagnosed with Autism Spectrum Disorder (ASD) and intellectual disability, aged seven to fourteen. The sequence of the procedure was: tests of rule following and tact-intraverbal in baseline; teaching with MEI for a spatial relation; final test of rule following and tact-intraverbal for the spatial relation not directly taught; generalization test. Multiple probes design coupled with multiple baseline across participants design was used. The results suggest that teaching with MEI was sufficient for the emergence of responses not directly taught and generalization. It could be inferred that the procedure was effective and efficient, as it generated the emergence of responses not directly taught and the learning took place in a few blocks.
\end{abstract}

KEYWORDS: Language. Multiple Exemplar Instruction. Autism.

\section{INTRODUÇÁo}

A manifestação dos sintomas centrais do Transtorno do Espectro do Autismo (TEA), como déficits na comunicação, na interação social e nos interesses restritos, pode variar ampla-

\footnotetext{
${ }^{1}$ https://doi.org/10.1590/1980-54702021v27e0228

${ }^{2}$ Os autores agradecem à Coordenação de Aperfeiçoamento de Pessoal de Nível Superior (CAPES) pelo apoio contínuo geral à pesquisa (PROEX 23038.005155 / 2017-67) e pela bolsa de Doutorado à primeira autora. Esta pesquisa foi conduzida como parte da tese de Doutorado da primeira autora junto ao Programa de Pós-Graduação em Educação Especial da Universidade Federal de São Carlos (UFSCar).

${ }^{3}$ Doutora pelo Programa de Pós-Graduação em Educação Especial. Universidade Federal de São Carlos (UFSCar). São Carlos / São Paulo / Brasil. E-mail: elacarvalhos@gmail.com. ORCID: https://orcid.org/0000-0003-1016-9718

${ }^{4}$ Doutor em Educação Especial e Professor do Departamento de Psicologia da Universidade Federal de São Carlos (UFSCar). São Carlos / São Paulo / Brasil. E-mail: nassim@ufscar.br. ORCID: https://orcid.org/0000-0003-4197-623X
} 